\title{
Bericht über eine in russischer Sprache erschienene dipterologische Arbeit.
}

\section{Von C. R. Osten-Sacken.}

In den Verhandlungen der naturforschenden Gesellschaft an der Universität Kharkoff, Vol. XV (auch separat, Kharkoff, 1881, pag. 9, $8^{\circ}$ mit 1 Tafel), hat Herr P. J. S te $p$ a $n$ off eine dipterologische Arbeit in russischer Sprache publicirt, welche den Titel $O$ prevrastcheniach $u$ dvukrylich semeistva Bombylidae (Ueber die Verwandlungen der Zweiflügler aus der Familie der Bombyliden) führt. Nachdem diese Schrift in entomologischen Kreisen weniger bekannt sein dürfte, so scheint es nicht ohne Interesse zu sein, wenn ich hierüber an diesem Orte berichte.

Es ist merkwürdig, dass in denselben Jahren $1879-1880$ mehrere Beobachter, ganz unabhängig von einander, über das Vorkommen von Bombyliden-Larven in den Eiersäcken von Wanderheuschrecken berichtet haben. Die Beobachtungen von L e m $\mathrm{m}$ o $\mathrm{n}$ und Riley in Nord-Amerika und von J. C a l ve rt an den Dardanellen sind bekannt und in den jährlichen entomologischen Berichten erwähnt worden; die von Herrn St e p a n oft hingegen sind bis jetzt unbeachtet geblieben. Er hat das zahlreiche Vorkommen der Larve eines Bombyliden, den er als Systoechus leucophaeus Meig., mit einem Fragezeichen, bestimmt, in den Eiersäcken der Heuschrecke Stauronotus vastator Stev. *) beobachtet. Die Larve hatte er schon früher (l. c., Vol. XIII) beschrieben; die Puppe ist in der gegenwärtigen Arbeit abgebildet. Gleichwie in Nord-Amerika mit Systoechus oreas zugleich auch ein Anthracide (Triodites mus) in den erwähnten Eiersäcken hospitirend gefunden wurde, so auch in Südrussland neben Systoechus leucophaeus M.? der Mulio obscurus Meig., dessen Puppe bei Herrn Stepan off ebenfalls abgebildet ist. Die Analogie geht aber noch weiter: in Nord-Amerika entdeckte Riley eine den Eiersäcken sehr schädliche Anthomyia-

*) Nach der Nomenclatur in Brunner v. Wattenwyl's Prodromus der europäischen Orthopteren würde die Art Stauronotus maroccanus Thunb (Syn. vastator Fischer) heissen. Ich muss aber zugleich darauf aufmerksam machen, dass Herr Stepa n off, Köppen's Meinung folgend, den St.vastator von St. cruciatus Charp. unterscheidet, während diese Arten von Brunner als Synonyma angeführt werden.

„Wiener Entomologische Zeitung“ IV. (15. Jänner 1885). Heft 1. 
Larve, deren Fliege er zuerst für eine Varietät der $A$. radicum hielt, welche aber später von Herrn Meade als $A$. angustifrons $\mathrm{M}$. bestimmt wurde. Herr S t e p a n off hat an Eiersäcken von Pachytylus migratorius das Vorkommen einer AnthomyiaLarve constatirt, deren Fliege er ebenfalls als $A$. radicum bestimmte. In Amerika wurde von jener Anthomyia in gewissen Localitäten ein Zehntel der Eier der Wanderheuschrecke (Caloptenus spretus) zerstört!

\section{0tiorrhynchus (Tournieria) Starcki n. sp.}

\section{Von 0. Retowski in Theodosia (Krim).}

\section{Otiorrhynchus Starcki n. sp.}

Oblongo-ovatus, piceus, pilis brevibus griseo-testaceis dense obsitus; rostro capite parum angustiore et non longiore, carinato, fronte oculo latiore; antennis longis, funiculi articulo secundo primo sesqui longiore, externis latitudine longioribus ; thorace longitudine paulo latiore, lateribus rotundato, post medium latissimo, confertim granulato; elytris ovalibus, seriatim punctatis, interstitïs subtiliter rugosis; femoribus anticis dente valido armatis, posticis muticis; tibiis anticis arcuatis, muticis. Long. $7^{1 / 2}-8 \mathrm{~mm}$. Patria : Caucasus occidentalis (Novorossisk).

Diese von meinem Freunde, Herrn Starck, bei Novorossisk in Mehrzahl gesammelte Species gehört der Bildung der Fühlerfurche nach, sowie durch ihre bauchigen, kurzeiförmigen, nach hinten verschmälerten Flügeldecken und den sehr grossen dreieckigen Zahn der Vorderschenkel in die fünfte Rotte des Subgenus Tournieria (Best. Tab. der europ. Coleopt. IX. Curculionidae v. Dr. G. Stierlin, p. 19). Von den fünf zu dieser Rotte gehörigen Species unterscheidet sie sich jedoch hinlänglich, um als besondere Art angesprochen zu werden. Drei Species: creticus Stl., decoratus Stl., und Reitteri Stl. sind, um nur die Hauptunterschiede hervorzuheben, beschuppt, während 0 . Starcki nicht beschuppt und dicht kurz behaart ist. Von den beiden ebenfalls behaarten Arten der Gruppe, brachialis Schh. und longipes Stl., zu denen $O$. Starcki als nächst verwandt zu stellen ist, unterscheidet sich dieser vornehmlich durch die ziemlich stark gekrümmten Vorderschienen, die Form des Halsschildes und die unbewehrten Hinterschenkel, sowie durch die rein schwarze Färbung des Körpers. 


\section{$2 \mathrm{BHL}$ Biodiversity Heritage Library}

Osten-Sacken, C. R. 1885. "Bericht über eine in russischer Sprache erschienene dipterologische Arbeit." Wiener entomologische Zeitung 4, 9-10. https://doi.org/10.5962/bhl.part.20090.

View This Item Online: https://www.biodiversitylibrary.org/item/44101

DOI: https://doi.org/10.5962/bhl.part.20090

Permalink: https://www.biodiversitylibrary.org/partpdf/20090

\section{Holding Institution}

Smithsonian Libraries

\section{Sponsored by}

Smithsonian

\section{Copyright \& Reuse}

Copyright Status: NOT_IN_COPYRIGHT

This document was created from content at the Biodiversity Heritage Library, the world's largest open access digital library for biodiversity literature and archives. Visit BHL at https://www.biodiversitylibrary.org. 